\title{
Housing influence on multi-band directional MEMS microphones inspired by Ormia ochracea
}

\author{
R. Bauer ${ }^{1}$, Y. Zhang ${ }^{1}$, J. C. Jackson ${ }^{1}$, W. M.Whitmer ${ }^{2}$, W. O. Brimijoin ${ }^{2}$, M. Akeroyd ${ }^{2}$, D. Uttamchandani ${ }^{1}$, \\ and J. F. C. Windmill ${ }^{1}$ \\ ${ }^{1}$ Department of Electronic \& Electrical Engineering, University of Strathclyde, Glasgow, UK \\ ${ }^{2}$ Scottish Section, MRC/CSO Institute of Hearing Research, Glasgow, UK \\ Email: ralf.bauer@strath.ac.uk
}

\begin{abstract}
A new bio-inspired multi-band directional MEMS microphone based on the hearing properties of the fly Ormia ochracea is presented, together with the behavioral influence of 3D-printed housings. The multi-user foundry fabricated microphone operates on four frequency bands, all below $10 \mathrm{kHz}$ and acts as a pressure gradient directional microphone with figure of eight polar pattern, or as an omni-directional microphone depending on the housing. The influence of an open or closed backside housing on the frequency response and directionality is shown, leading to the loss of directionality with no acoustic access to the backside of the fly-ear inspired microphone membrane.
\end{abstract}

Keywords-MEMS directional microphone; bio-inspired; 3Dprinted housing; multi-band sensing; piezoelectric sensing

\section{INTRODUCTION}

The use of Microelectromechanical Systems (MEMS) miniature microphones in consumer products such as smart phones is ever increasing, helped by the use of multiple devices for noise cancellation or determination of sound origins. A different approach for achieving a directional sensor has shown increasing research interest over the last decade, focusing on bio-inspired designs of a single microphone with further targeted application in e.g. hearing aids. The design inspiration originates from the fly Ormia ochracea which has an exceptional sound localisation ability through using the combination of symmetric and asymmetric resonance modes of its coupled tympanic membranes to extract directionality cues. Multiple MEMS microphones based on this principle have been presented since Miles et al.'s initial publication [1], with capacitive comb-drive sensing [2, 3], piezoelectric sensing [4],

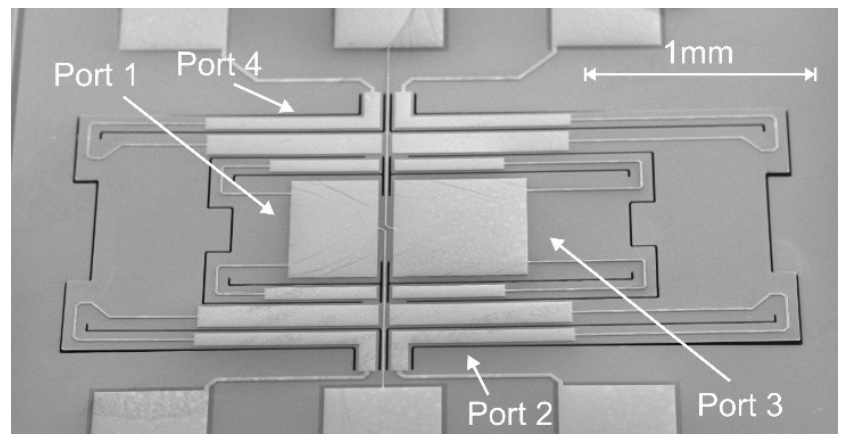

Fig. 1: SEM of fabricated device.

The work was supported by the Engineering and Physical Sciences Research Council (EPSRC) under grant EP/M026701/1, and by the European Research Council under the European Union's Seventh Framework Programme (FP/2007-2013) / ERC Grant Agreement n. [615030]. or optical read out $[1,5]$. While the fly's hearing focuses on a single frequency with exceptional directionality resolution, this high Q resonance behaviour has a low electrical response off its main resonances, as shown in the publications mentioned above. In our previous work we expanded the two original movement resonance modes to a multi-band operation through multiple membranes set in each other, with operating frequencies located below $15 \mathrm{kHz}[6]$.

In the work presented here, this design has been adapted for higher electrical signal outputs of the aluminium nitride (AIN) piezoelectric sensing while simultaneously moving the operational frequency bands to below $10 \mathrm{kHz}$ to operate in the audible range relevant to human speech recognition. This new design is also used to investigate the influence of different 3Dprinted housings and corresponding sound access to the back of the microphone membrane, specifically studying changes to the directionality of the acoustic response, as previous works have only studied the frequency and directionality behaviour of unhoused devices.

\section{MICROPHONE DESIGN AND FABRICATION}

\section{A. Piezoelectric mulit-band MEMS microphone}

The multi-band silicon MEMS microphone is based on our previous work [6] and work by Kuntzman et al. [7], using in our case AlN as a piezoelectric sensing layer. The overall dimensions of the fabricated device shown in Fig. 1 are $3.2 \mathrm{~mm}$ $\mathrm{x} 1.7 \mathrm{~mm}$ for the outer membrane and $2 \mathrm{~mm} \times 0.9 \mathrm{~mm}$ for the inner membrane, which are responsible for the first two, and third and fourth resonance modes of interest respectively. Both the outer and inner membranes have $250 \mu \mathrm{m}$ x $20 \mu \mathrm{m}$ torsion beams and two $100 \mu \mathrm{m}$ wide bending cantilevers anchored at the rear of the membrane to increase the stress induced piezoelectric signals. The active sensing areas are split into 6 ports, 4 on the outer membrane and 2 on the inner membrane (see Fig. 1).

\section{B. Multi-user fabrication process}

The devices are fabricated using the commercially available multi-user process PiezoMUMPS, offered by Memscap Inc. The single crystal silicon device layer is $10 \mu \mathrm{m}$ thick, with a $400 \mu \mathrm{m}$ thick substrate fully backside etched to release the moving device parts. The $500 \mathrm{~nm}$ thick piezoelectric AlN layer is deposited using reactive sputtering, with a $1 \mu \mathrm{m}$ thick layer of Al allowing electrical connections to the sensing areas. A 


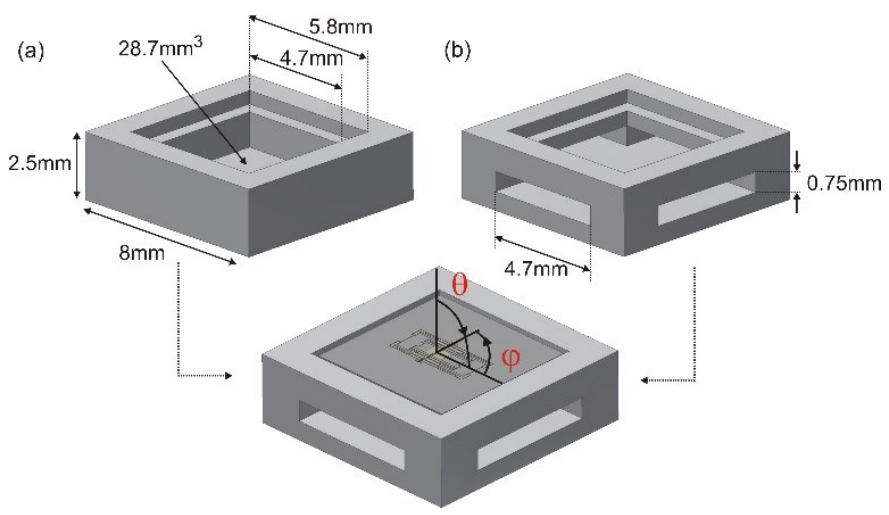

Fig. 2: 3D-printed housings with dimensions for (a) closed backside cavity and (b) open backside cavity.

$200 \mathrm{~nm}$ thermal oxide allows isolation of the AlN connections to the doped silicon, which forms the second electrical port for sensing the generated piezoelectric voltages. The overall MEMS chip size is $5.5 \mathrm{~mm} \times 5.5 \mathrm{~mm}$, with the microphone design placed at its centre.

\section{Microphone housing design and fabrication}

To evaluate the influence of a backside air cavity and access of sound to the rear of the microphone membrane, two housing variations have been designed and fabricated using rapidprototyping 3D-printing (see Fig. 2). The housings have a footprint of $8 \mathrm{~mm} \times 8 \mathrm{~mm}$ with a height of $2.5 \mathrm{~mm}$. An acoustic backside cavity with a volume of $28.7 \mathrm{~mm}^{3}$ is either fully sealed through the MEMS chip (see Fig. 2(a)) or has four $4.7 \mathrm{~mm} \mathrm{x}$ $0.75 \mathrm{~mm}$ sound access ports (see Fig. 2(b)). The housings are fabricated using a $25 \mu \mathrm{m}$ resolution 3D-printer (EnvisionTec Desktop Aureus) which is based on photo-polymerisation of a liquid resin (R11 by EnvisionTec). Electrical connections are made through wire bonding between the MEMS device and metal insets in the 3D-printed part.

\section{METHODS}

\section{A. Finite Element modelling}

The finite element modelling tool COMSOL Multiphysics is used to predict the mechanical behaviour and acoustic frequency response of the MEMS microphones. A solid-mechanics Eigenfrequency analysis is constructed to monitor the Eigenmode shapes using the anisotropic material properties of silicon. The thin AIN layer is included for this evaluation. To model the frequency response to an incident soundwave, an acoustic-solid-interaction model is constructed, including a spherical air domain around the device, chip and holder. A plane wave sound source with $1 \mathrm{~Pa}$ sound pressure is defined, with the sound travel direction normal to the membrane. The model does in this case not include the AlN layer in order to reduce computational requirements through the high aspect ratios involved with the $500 \mathrm{~nm}$ thick AlN layer.

\section{B. Experimental setup}

The device response in the different housings is characterised for both the frequency and directional response using a single frequency sound excitation sweep. The devices are mounted on a rotation stage placed inside an anechoic

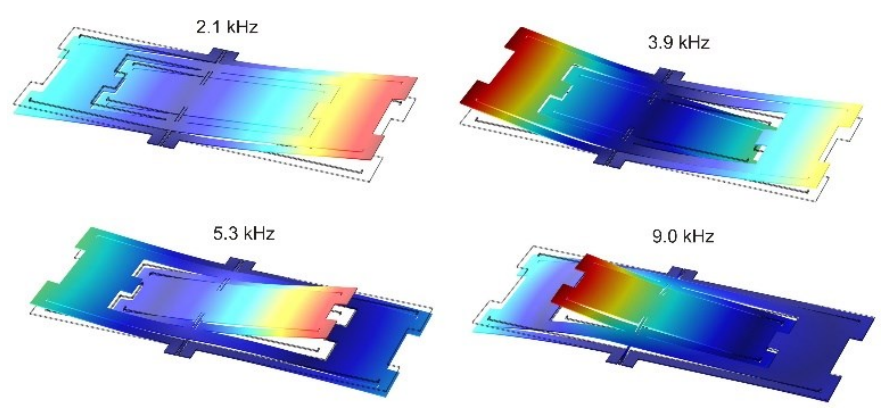

Fig. 3: Eigenmode shapes and frequencies of the multi-band bio-inspired microphone design.

chamber, with the exciting speaker (Visaton FRS5) placed at a distance of $1 \mathrm{~m}$ from the device. The speaker is directly driven by an Agilent 33220A signal generator with $10 \mathrm{~V}_{\mathrm{pp}}$ amplitude. The electrical signals are picked up through a pre-amplifier with $56 \mathrm{~dB}$ gain and then filtered through a Stanford Research Systems SR 850 lock-in amplifier with a further $26 \mathrm{~dB}$ gain and $0.78 \mathrm{~Hz}$ filter bandwidth, before being recorded through a Tektronix TDS1002B Oscilloscope. 3D-printed mounting adapters fix the housings in the experimental setup to allow rotational scans along the polar $(\theta)$ and azimuthal $(\varphi)$ coordinates of the microphone.

\section{SimUlated AND EXPERIMENTAL RESUlTS}

\section{A. Frequency response}

The simulated Eigenmode shapes and -frequencies of the multi-band MEMS microphone are shown in Fig. 3. The first two frequencies $(2.1 \mathrm{kHz}$ and $3.9 \mathrm{kHz})$ depict the outer membrane movement changing from a rocking motion to a bending motion, with the inner membrane rocking in both cases. The third and fourth frequencies $(5.3 \mathrm{kHz}$ and $9.0 \mathrm{kHz}$ respectively) show an out-of-phase rocking and bending of the two membranes. The simulation results of the mechanical response to a plane-wave, single frequency, sound excitation is shown in Fig. 4, comparing the response of the two housing variations. The displacement amplitude of the microphone is measured at the outer edge of the membrane associated with port 4, with frequency steps of $100 \mathrm{~Hz}$. The simulations show a higher mechanical response with the open housing, with all four frequencies clearly present. The resonance frequencies are lower than the calculated Eigenfrequencies due to the missing AlN

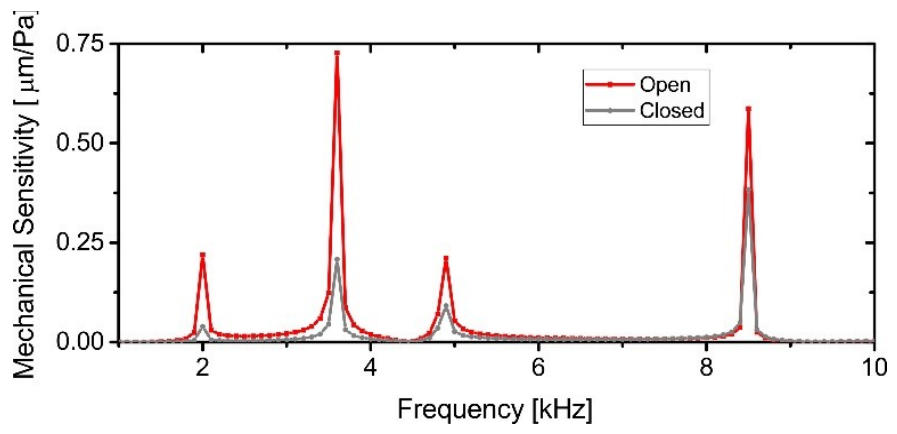

Fig. 4: Simulated mechanical frequency response at normal incidence for closed and open backside holder. 


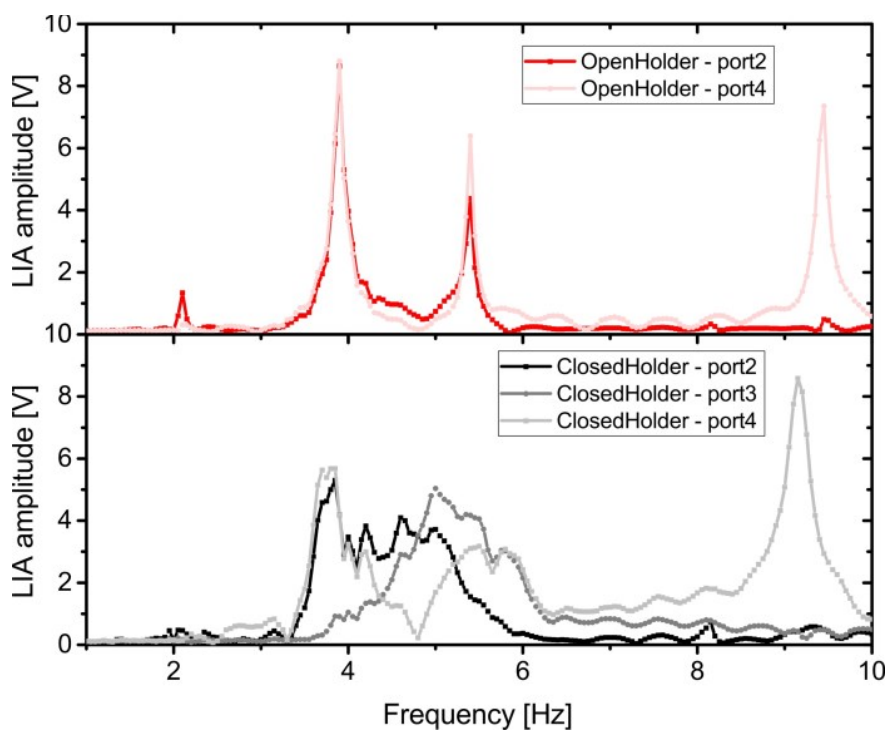

Fig. 5: Experimental electrical frequency response at normal incidence for closed and open backside holder.

layer in the acoustic response simulation. Damping was only considered in the form of pressure-acoustic damping.

The experimental characterisation of the frequency response of both device-holder combinations is presented in Fig. 5, showing the lock-in amplifier (LIA) output with varying frequency. The measurement ports 2 and 4 are used to recover the highest possible electrical signal for all frequencies. The electrical signal frequency response of the open holder (Fig. 5, top) shows good agreement with the simulation, with the measured Eigenfrequencies being at $2.1 \mathrm{kHz}, 3.9 \mathrm{kHz}, 5.4 \mathrm{kHz}$ and $9.45 \mathrm{kHz}$. The response between the second and third resonance is non-zero, which is hinted at by the simulation, with the $2.1 \mathrm{kHz}$ response being lower than expected. For the closed holder (Fig. 5, bottom) the electrical signal frequency response shows a widening of all frequency bands, with a vanishing response at $2.1 \mathrm{kHz}$ but a broadband response between the second and third resonance. The highest frequency shows the expected resonance peak, however still broadened compared to the response of the open holder. The significant increase in bandwidth is believed to originate from damping in the closed backside cavity, however only little amplitude reduction is present for this damping case which is currently under further investigation.

\section{B. Directionality}

The measured directivity patterns in polar $(\theta)$ direction are shown for both holder combinations in Fig. 6 , with $0^{\circ}$ depicting the excitation normal to the membrane. The polar response of the holder with open backside shows a figure of eight pattern expected from a pressure gradient microphone for the three highest frequencies, with bias to the front and back at $9.45 \mathrm{kHz}$ and $3.9 \mathrm{kHz}$ respectively. The lowest frequency $2.1 \mathrm{kHz}$, however, shows a strong bias to the front. For the closed holder the same measurement shows an omni-directional response for all four frequencies, with only the highest frequency at $9.2 \mathrm{kHz}$ having a small bias towards the front side of the membrane.

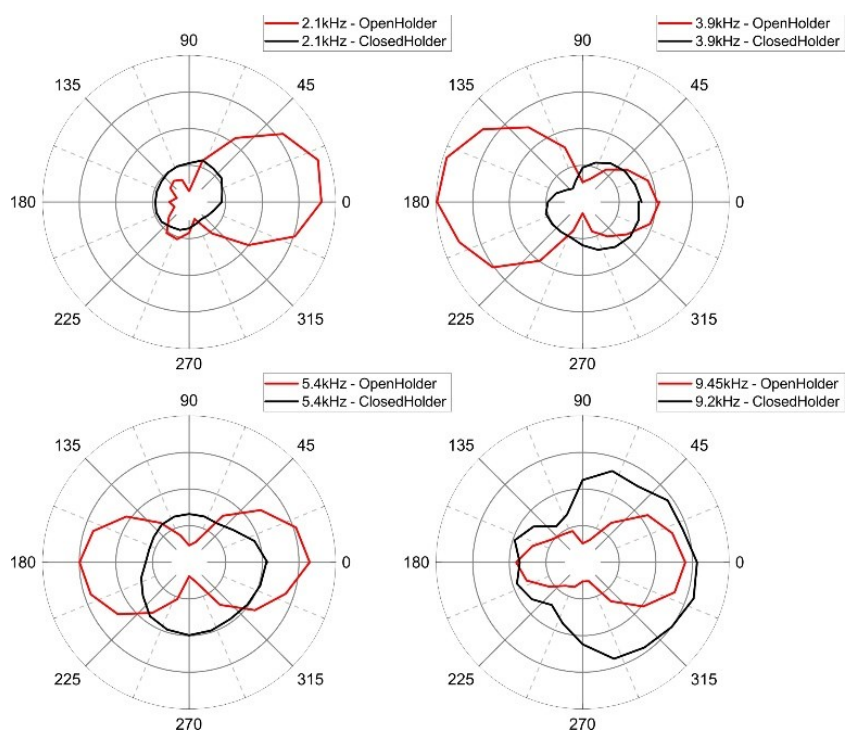

Fig. 6: Experimental measurement of the directional response in $\theta$ for the four frequency bands for the closed and open backside holder.

\section{DisCUSSION AND CONCLUSION}

Both the frequency response and the polar directionality behaviour of the open backside holder show a fit between the measurements and simulation. The closed holder frequency response however shows a broader frequency response experimentally than predicted. Its directionality in $\theta$ shows nevertheless an omni-directional response as expected according to initial simulations. Further investigations in the broadening of the frequency response without significant amplitude drop are currently ongoing and will be presented. A clear influence of the housing and packaging of the new multiband piezoelectric microphone is shown in the presented investigation, with access to the backside required to follow a first order directional behaviour.

\section{REFERENCES}

[1] R. N. Miles, et al. "A low-noise differential microphone inspired by the ears of the parasitoid fly Ormia ochracea.," J. Acoust. Soc. Am., vol. 125, no. 4, pp. 2013-2026, 2009.

[2] R. N. Miles, W. Cui, Q. T. Su, and D. Homentcovschi, "A MEMS LowNoise Sound Pressure Gradient Microphone With Capacitive Sensing," J. Microelectromechanical Syst., vol. 24, no. 1, pp. 241-248, 2015.

[3] M. Touse, J. Sinibaldi, and G. Karunasiri, "MEMS directional sound sensor with simultaneous detection of two frequency bands," Proc. IEEE Sensors, pp. 2422-2425, 2010.

[4] M. L. Kuntzman, N. N. Hewa-Kasakarage, A. Rocha, D. Kim, and N. A. Hall, "Micromachined In-Plane Pressure-Gradient Piezoelectric Microphones," IEEE Sens. J., vol. 15, no. 3, pp. 1347-1357, 2015.

[5] A P. Lisiewski, H. J. Liu, M. Yu, L. Currano, and D. Gee, "Fly-ear inspired micro-sensor for sound source localization in two dimensions.," J. Acoust. Soc. Am., vol. 129, no. 5, pp. EL166-L171, 2011.

[6] Y. Zhang, R. Bauer, J. F. C. Windmill, and D. Uttamchandani, "Multiband asymmetric piezoelectric MEMS microphone inspired by the Ormia ochracea," in 2016 IEEE 29th International Conference on Micro Electro Mechanical Systems (MEMS), 2016, pp. 1114-1117.

[7] M. L. Kuntzman and N. A. Hall, "Sound source localization inspired by the ears of the Ormia ochracea," Appl. Phys. Lett., vol. 105, no. 3, p. 033701, 2014. 